\title{
Évolution du littoral de la côte des Landes et du Pays Basque au cours des dernières années*
}

\author{
Behaviour of the French atlantic coastline in the Landes \\ and Basque regions during recent years
}

\author{
Claude Migniot et Jacques Lorin
}

Laboratoire Central d'Hydraulique de France

\section{Introduction}

Entre l'embouchure de la Gironde et la frontière d'Espagne le littoral du Golfe de Gascogne s'étend sur près de 260 kilomètres, les falaises rocheuses et découpées du Pays Basque apportant un contraste avec les grandes plages sableuses situées au Nord de l'Adour.

Le long de ce littoral la rivière de l'Adour offre une discontinuité entre les forêts landaises avec leur frange de lacs et d'étangs et l'intérieur du Pays Basque avec toute la douceur et le charme de ses collines rehaussées au loin par le chaine des Pyrénées.

Vers le large les fonds marins se rapprochent d'autant plus de la côte que 1'on descend plus au Sud du Golfe et si les profondeurs de $100 \mathrm{~m}$ sont à près de $85 \mathrm{~km}$ au large de la pointe de Grave, elles ne sont plus qu'à $12 \mathrm{~km}$ au droit de Biarritz. De même la pente moyenne des fonds entre l'estran et les petits fonds de $-20 \mathrm{~m}$ passe de $0,3 \%$ en extrême Nord du littoral à près de $1 \%$ dans le Sud. Le gouf de Capbreton, véritable entaille géologique de plus de $500 \mathrm{~m}$ de profondeur que l'on retrouve perpendiculairement à la côte, va apporter une forte perturbation dans la bathymétrie. (Figure 1)

Cette configuration générale du littoral du Golfe de Gascogne, qui s'ouvre vers l'Ouest et le. NW sur une distance pratiquement infinie lui donne une place privilégiée par la puissance de ses houles ; c'est d'ailleurs sur cette partie de nos côtes françaises que l'on rencontre les vagues les plus fortes et, de ce fait, les pouvoirs de destruction les plus importants.

Associée à un déficit sédimentaire, cette force des houles entraine une dégradation progressive des fonds marins et, en certains points, un recul spectaculaire de

(*) Exposé présenté à Bayonne le 28 octobre 1978. Les études faites pour la MIACA apporteront des précisions à cette étude préliminaire.

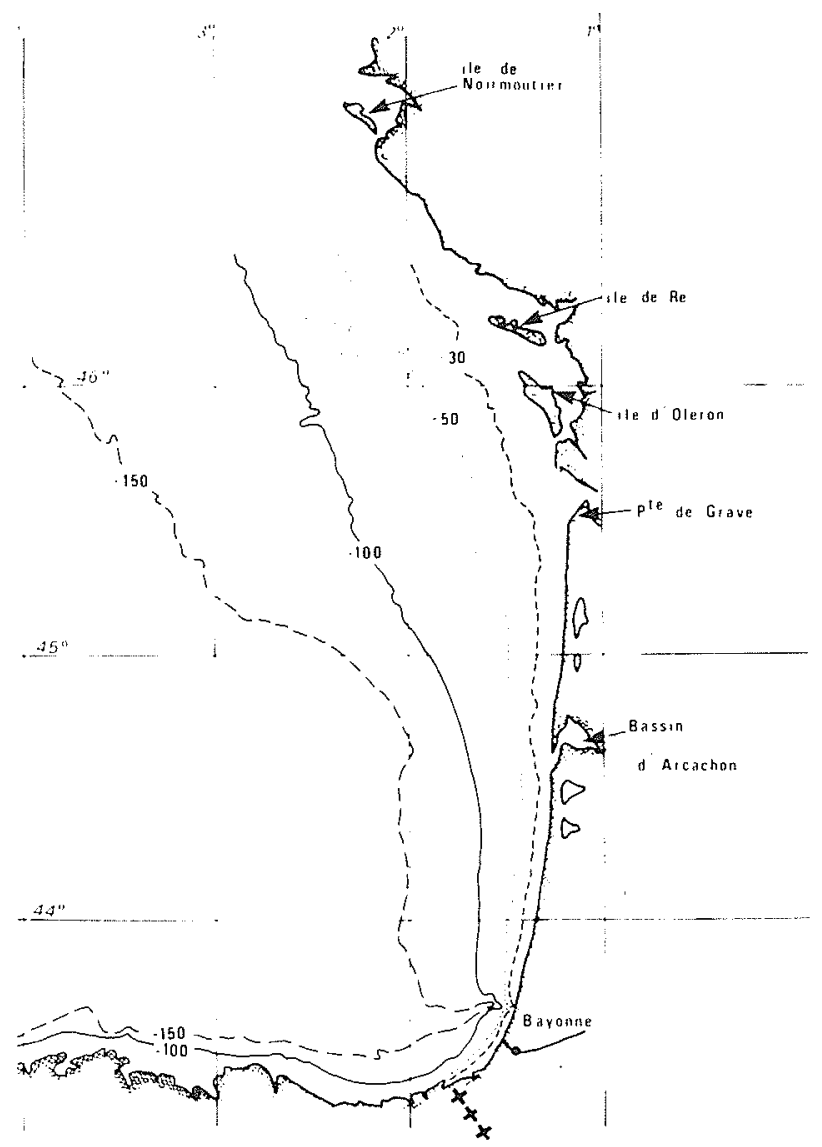

Figure 1 - Carte des fonds du Golfe de Gascogne

la ligne de côte qui détériore progressivement l'un des plus beaux patrimoines touristiques de notre pays.

Devant l'ampleur des phénomènes naturels inéluctables, exacerbés dans certains secteurs par les inter- 
ventions humaines multiples allant depuis les emprunts de sable jusqu'aux ouvrages d'amélioration locale des plages ou des accès aux ports, la Mission d'Aménagement de la Côte d'Aquitaine (M.I.A.C.A.) a demandé au Laboratoire Central d'Hydraulique de France (L.C.H.F.) d'examiner l'importance du recul du littoral au cours des dernières années.

Pour mieux situer ce problème nous rappellerons tout d'abord les phénomènes géologiques très récents qui ont contribué aux modifications de la ligne du rivage du Golfe de Gascogne.

L'importance des phénomènes océanographiques, et plus particulièrement des houles, sera ensuite donnée ainsi que le mécanisme des mouvements sédimentaires en résultant.

Des exemples précis du recul du littoral au cours du dernier siècle permettront de mieux quantifier les érosions, que ce soit entre Soulac et la Pointe de Grave, devant Arcachon, aux abords de Cap Breton, près de l'embouchure de l'Adour ou le long des falaises de Biarritz et de la Baie de Saint Jean de Luz.

A l'échelon des dernières décennies, des dernières années, il sera possible enfin, à l'aide de quelques photographies qui viennent d'être réalisées, de visualiser l'évolution de la côte des Landes et du Pays Basque et de se faire une idée plus générale des phénomènes que l'on attribue quelquefois seulement à des points particuliers sur lesquels l'urbanisaton excessive de la zone côtière a at tiré l'attention.

\section{Rappel des phénomènes géologiques récents qui ont contribué aux modifications de la ligne de rivage du Golfe de Gascogne.}

$\mathrm{Au}$ cours du quaternaire, transgressions et régressions se sont succédées modifiant considérablement la surface des zones émergentes.

Si il y a 300000 ans le niveau des eaux était entre 50 et $100 \mathrm{~m}$ au-dessus du niveau actuel de la mer, il était à une époque récente -il y a seulement 15000 ans - à une cote 100 à $120 \mathrm{~m}$ plus basse. A partir de cette date le niveau marin remonte progressivement pour atteindre

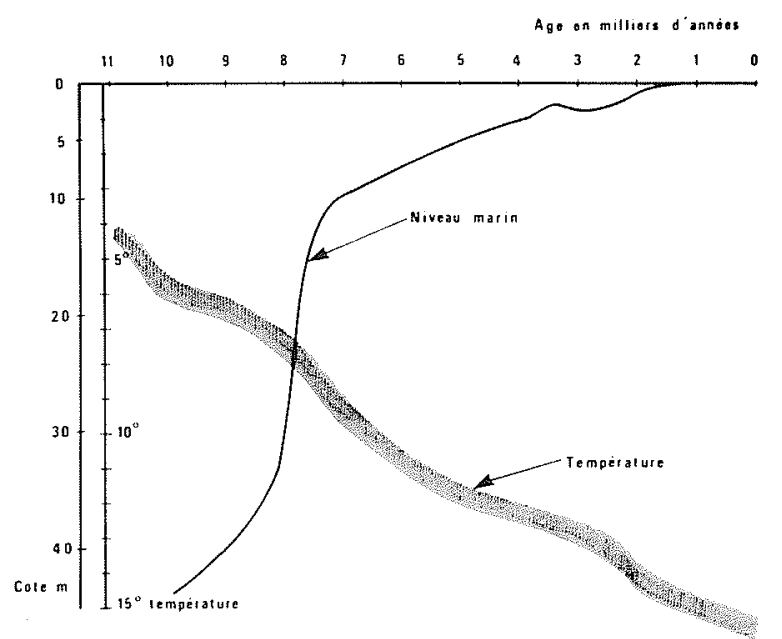

Figure 2 - Variation du niveau marin depuis 15000 ans. la cote $-10 \mathrm{~m}$ il y a 6000 ans environ et tendre vers le moyen âge à une certaine stabilité après être monté légèrement plus haut que la cote actuelle et être redescendu (transgression Dunkerquienne il y a environ 1000 ans). Dans l'état actuel le niveau marin remonterait très lentement à raison de 1 à $1,5 \mathrm{~mm}$ par an. (Figure 2).

La position du littoral de la côte de Gascogne a suivi cette remontée des eaux et, progressivement, des étendues de près de $80 \mathrm{~km}$ de largeur se sont trouvées submergées au cours de la transgression flandrienne (Figure 3).

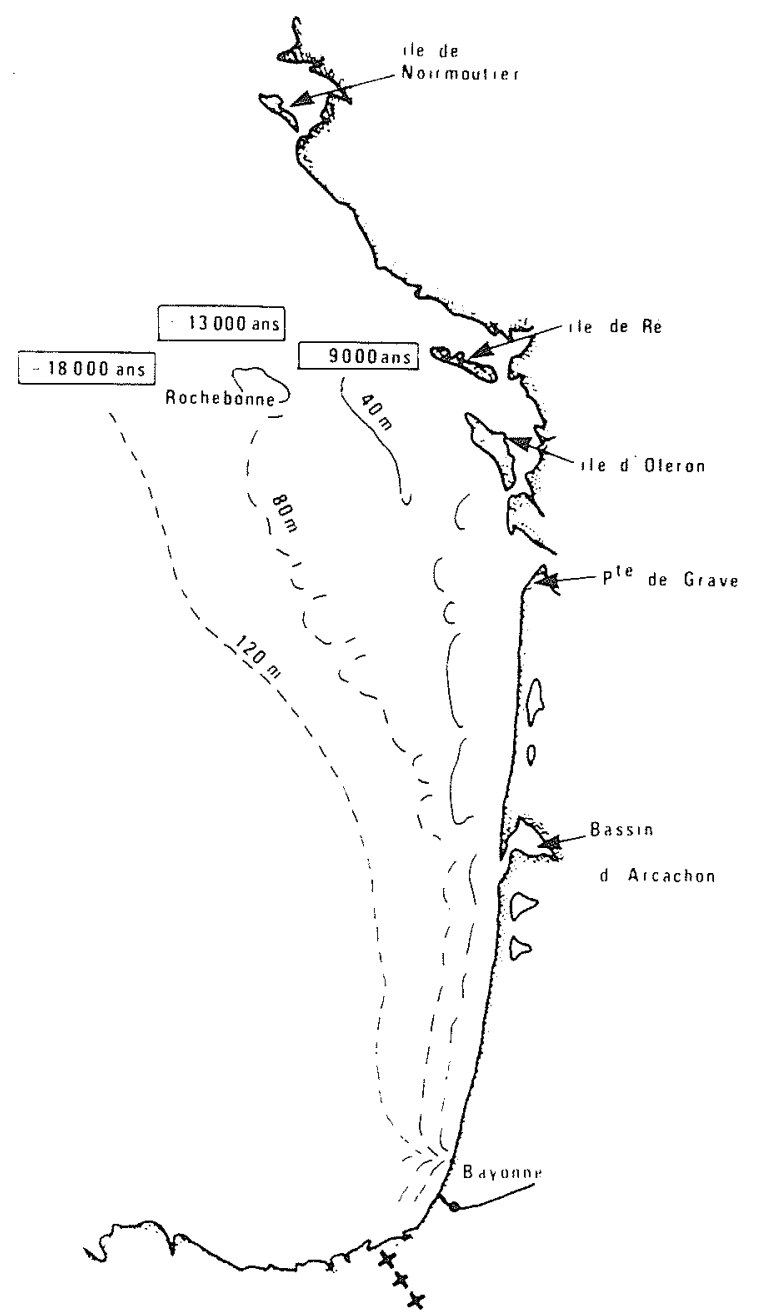

Figure 3 - Différentes positions du littoral au cours de la transgression flandrienne.

Parallèlement ă ces vastes fluctuations du niveau des eaux les terres émergées peuvent, en certains points, subir des surélévations ou des abaissements susceptibles de modifier la position de la ligne de rivage. Pour de nombreux géologues la tendance générale serait à un abaissement très lent des terres le long du Golfe de Gascogne.

Sur ce vaste plateau continental finalement livré à l'action des eaux, les houles pouvaient trouver un stock sédimentaire important à remanier, à remonter vers le littoral pour l'alimenter. Depuis plus d'un millier d'années ce stock s'épuise progressivement réduisant une source d'apports sédimentaires. 
Par ailleurs on peut penser que l'activité tectonique actuelle puisse aller, en certains secteurs géographiques précis, à l'encontre de la stabilité du littoral.

\section{L'importance des phénomènes océanographiques et plus particulièrement des houles}

Les houles du Golfe de Gascogne qui remanient les fonds sont parmi les plus fortes que l'on puisse rencontrer le long du littoral français et les courbes de probabilité montrent que, pendant 50 jours par an, l'amplitude $\mathrm{H} 1 / 10$ (correspondant à l'amplitude la plus forte toutes les 10 vagues) dépasse $4 \mathrm{~m}$, pendant 10 jours $5,70 \mathrm{~m}, 2$ jours par an 7,50 m et un jour tous les 10 ans elle peut atteindre $11 \mathrm{~m}$. La tempête du 2 Décembre 1976 a donné des amplitudes maximales de $12,30 \mathrm{~m}$ et de $16 \mathrm{~s}$ de période qui montrent bien toute la puissance destructrice que ces houles peuvent avoir (Figure 4).

La marée avec une variation du niveau de l'eau pouvant atteindre $4,50 \mathrm{~m}$ par vives eaux exceptionnelles sera le deuxième paramètre qui interviendra dans l'évolution $\mathrm{du}$ littoral. Cette marée pourra non seulement modifier la cote d'action des houles mais engendrer des pénétrations et des sorties d'eau importantes dans les rivières ou les bassins. Dans l'Adour le volume oscillant qui pénètre et sort à chaque marée peut atteindre en vives eaux 70 millions de $\mathrm{m}^{3}$; il est de 400 millions de $\mathrm{m}^{3}$ dans le Bassin d'Arcachon et atteint 3 milliards de $\mathrm{m}^{3}$ en Gironde

De même les débits des rivières peuvent remanier les fonds, l'Adour ayant un débit de crue décennale de $2500 \mathrm{~m}^{3} / \mathrm{s}$ et pendant 5 jours par an elle dépasse 1000 $\mathrm{m}^{3} / \mathrm{s}$. Il ne faut pas, par contre, compter sur des apports de sédiments sableux ou graveleux par les rivières, les quantités étant négligeables soit par suite d'une puissance érosive trop faible, soit par suite de l'épuisement du stock sédimentaire disponible.

Les courants généraux et les courants de marée, en-dehors des abords des rivières ou bassins, sont faibles et inférieurs à $0,20 \mathrm{~m}$ par seconde. Ils sont à eux seuls incapables de transporter les sédiments par charriage

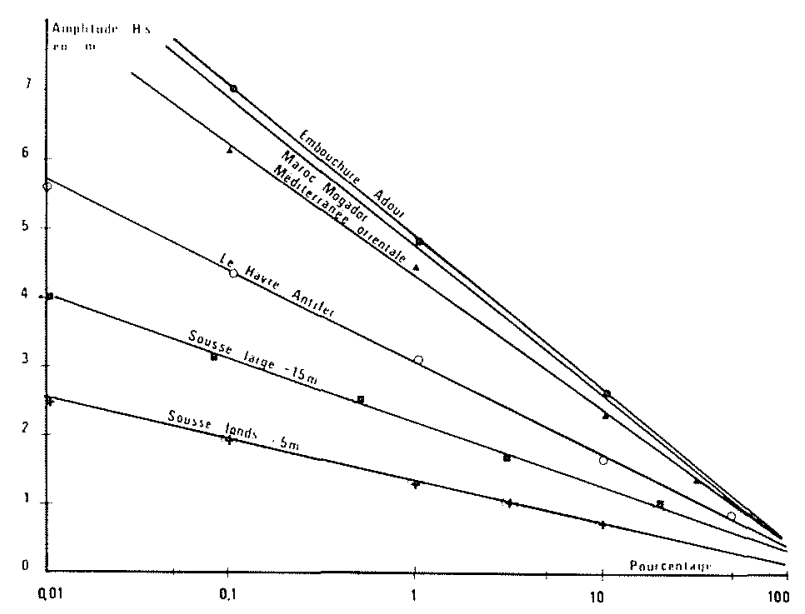

Figure 4 - Comparaison des houles du Golfe de Gascogne avec les autres points du monde mais peuvent remonter des matières en suspension ou en flottaison vers le Nord.

On ne saurait également négliger l'action des vents locaux qui en plus de leur influence sur la formation des vagues, peuvent entrainer des transports éoliens avec une remontée des sables vers les dunes.

\section{Les mouvements sédimentaires}

Si les sédiments des petits fonds marins sont constitués de sables fins de 0,10 à $0,20 \mathrm{~mm}$ de diamètre on trouve fréquemment sur les plages un mélange de plusieurs stocks sédimentaires comportant des éléments grossiers (madrague) mélangés à des sables plus fins. Sous le placage des sédiments fins du large on peut également retrouver des éléments grossiers sous une épaisseur de sable fin de 1,5 à $2 \mathrm{~m}$. Dans tous les cas la puissance sédimentaire des sables est très importante tout le long du littoral de la Côte des Landes.

Ces matériaux vont être soumis aux actions océanographiques et être remaniés, transportés soit perpendiculairement au littoral soit parallèlement.

Sous l'action des mouvements orbitaux de la houle, en effet, les fonds marins sont sollicités par des courants alternatifs importants, qui atteignent par exemple $2 \mathrm{~m} / \mathrm{s}$ par une profondeur de $10 \mathrm{~m}$ et par une houle de $5 \mathrm{~m}$ d'amplitude et $10 \mathrm{~s}$ de période, et subiront sur place une oscillation d'autant plus grande que la houle sera plus forte et les profondeurs plus faibles. Des formules permettent de calculer ces valeurs de la vitesse orbitale maximale sur les fonds. Connaissant par ailleurs la vitesse critique nécessaire pour provoquer le début d'entraînement d'un gain de sédiment il est possible d'avoir une bonne présomption sur la limite d'action des houles sur les fonds.

A ces mouvements d'oscillation se superposent au large des courants d'entraînement soit vers la côte, très près du fond et si la couche limite est laminaire, soit vers le large au-dessus des fonds et à mi-profondeur. Une particule solide pourra donc suivant le cas être remontée au rivage si elle est suffisamment lourde pour ne pas être remise en suspension, où être dispersée vers le large dans les courants de compensation si elle se détache suffisamment du fond. Ces échanges continucls contribuent au triage des matériaux dans la zone active de la houle.

L'importance de ces mouvements sédimentaires perpendiculairement au littoral a pu être calculée et mesurée en nature grâce à l'utilisation de traceurs radioactifs. On constate dans le cas du Golfe de Gascogne que ces mouvements dans le profil peuvent atteindre $500000 \mathrm{~m}^{3}$ par an et par kilomètre de plage, les volumes déplacés diminuant très rapidement avec la profondeur pour pratiquement s'annuler par des fonds de $-20 \mathrm{~m}$ (Figure 5).

Sous l'action des houles obliques un puissant courant littoral prendra également naissance à partir du point de déferlement des vagues. La vitesse de ce courant de houle, parallèle à la côte, pourra atteindre 1,5 à $1,75 \mathrm{~m} / \mathrm{s}$ en tempête et déplacera des masses d'eau considérables dont le débit dépasse parfois $2000 \mathrm{~m}^{3} / \mathrm{s}$. 


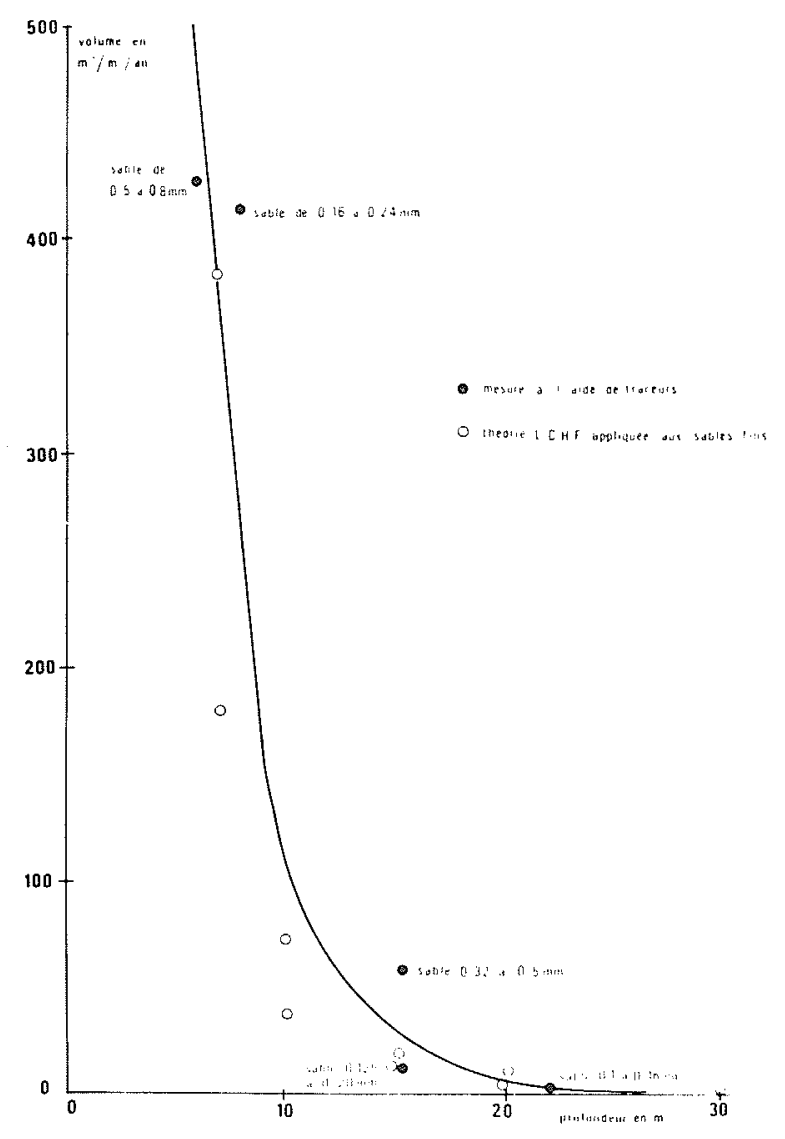

Figure 5 - Mouvements sédimentaires dans le profil le long de la côte des Landes.
Les sédiments brassés par les houles, soumis à ce courant, vont être entraînés parallèlement au littoral sur de grandes distances. On a évalué le transport résultant de sable du Nord vers le Sud à $800000 \mathrm{~m}^{3}$ par an devant Arcachon, à plus de $500000 \mathrm{~m}^{3}$ au Nord de Cap Breton et à 250000 à $300000 \mathrm{~m}^{3}$ au Nord de l'Adour. Au Sud de l'Adour et le long de la côte des Basques la résultante des mouvements tend à s'annuler mais les transits alternatifs du Nord vers le Sud et du Sud vers le Nord gardent toute leur puissance avec environ 400000 à $500000 \mathrm{~m}^{3}$ par an dans chaque sens. Dans tous les cas le maximum du transit littoral s'effectue par houle forte au voisinage de la zone de déferlement et, en beau temps, plus près de la côte en "dents de scie" sur l'estran. Les volumes déplacés varient comme le carré de l'amplitude et comme la période et sont donc 10 fois plus importants pour une houle de $3 \mathrm{~m}$ d'amplitude que pour une houle de $1 \mathrm{~m}$ et 70 fois plus importants pour une houle de $6 \mathrm{~m}$ d'amplitude (Figures 6 et 7).

Rappelons que les transports éoliens, qui remontent les sables vers les dunes, atteignent des valeurs beaucoup plus modestes que l'on évalue le long de la côte des Landes entre 15000 et $20000 \mathrm{~m}^{3}$ par an et par kilomètre de plage.

Remontée des sables sous l'action des houles, apports terrigènes des rivières, ont permis pendant de nombreuses décennies de maintenir un équilibre sédimen-. taire entre les transports, les triages avec échange vers le large, et une certaine stabilité des lignes de rivage. Actuellement les apports terrigènes sont pratiquement inexistants, les fonds marins s'épuisent progressivement
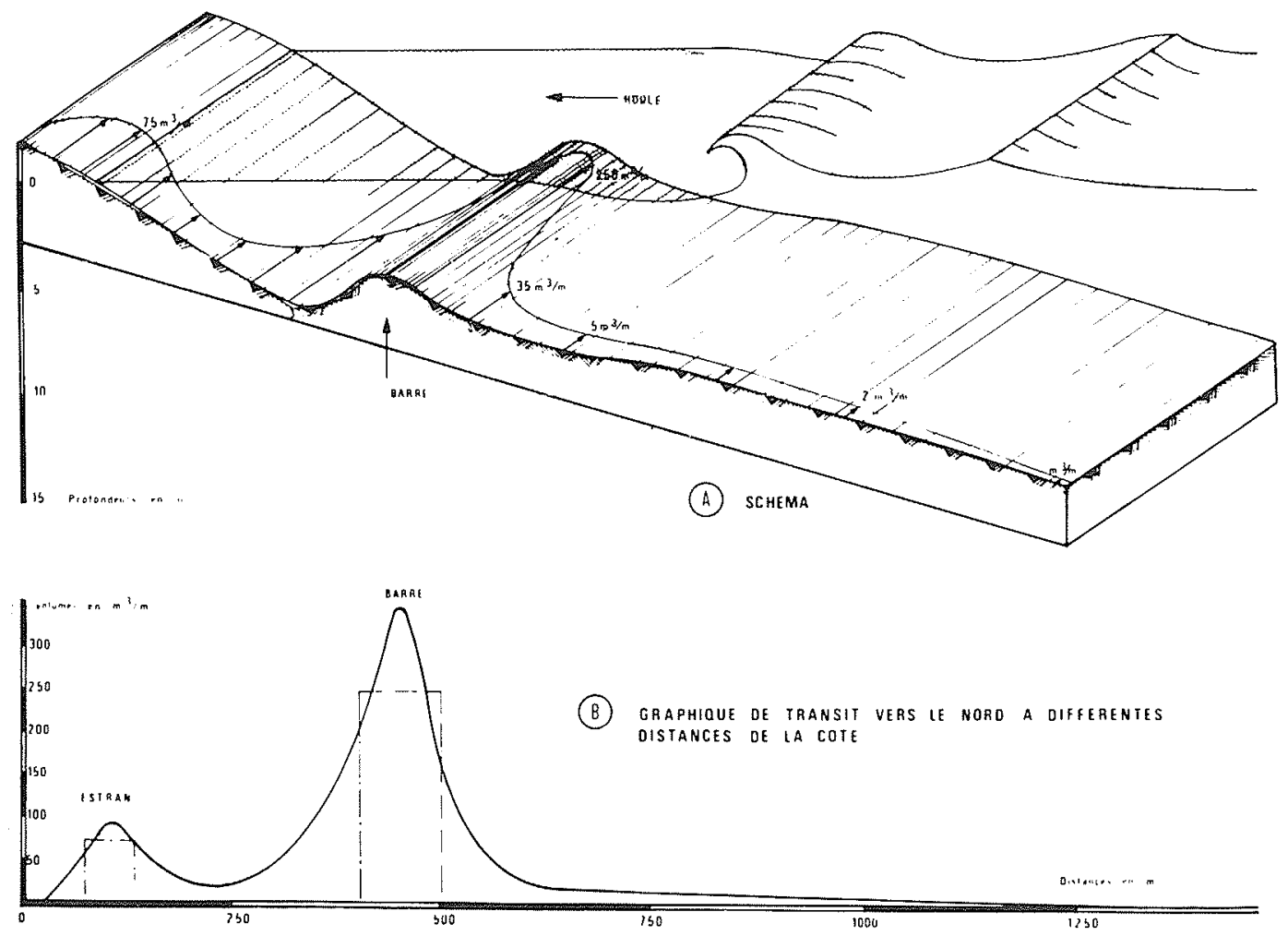

Figure 6 - Exemple des mouvements sédimentaires parallèles à la côte. 


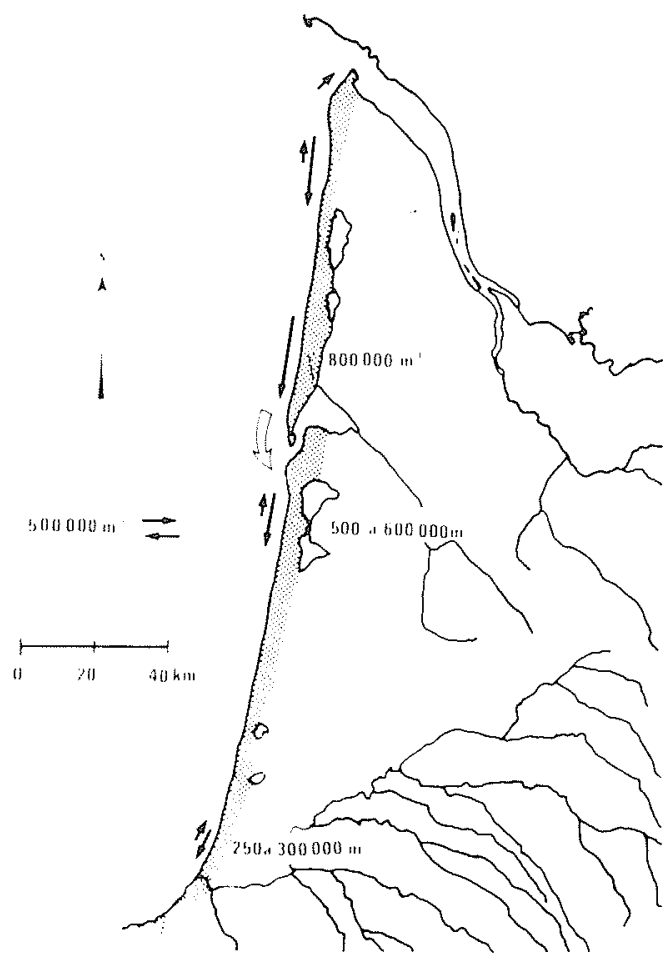

Figure 7 - Répartition du transit littoral le long de la côte des Landes.

et un déficit sédimentaire quasi général apparât le long des littoraux.

Les interventions humaines, qu'il s'agisse de protection locale d'un site, de l'amélioration de l'accès à un port, d'emprunts de sable ou de fixation de la ligne de côte, vont aggraver un équilibre déjà très précaire si ces interventions ne sont pas faites avec toutes les précautions désirables et en les remettant dans un cadre littoral très général. Qu'il suffise de rappeler que l'on extrait, chaque année, 125 à 150 millions de $\mathrm{m}^{3}$ d'agrégats (sables, graviers, galets) sur l'ensemble du territoire français pour se faire une idée de la réduction des apports terrigènes et du déficit littoral, même si une partie seulement de ces emprunts peut intervenir dans l'équilibre sédimentaire général fluvial et marin.

\section{Le recul du littoral de la côte des Landes et du Pays Basque au cours des deux derniers siècles}

Tous les auteurs, qu'ils soient géographes, géologues, géomorphologues, spécialistes des eaux et forêts, s'accordent à admettre que l'ensemble du littoral du Golfe de Gascogne, en-dehors de certains secteurs protégés par une couche d'alios ou de brie sur laquelle reposent les dunes, s'érode d'une façon à peu près continue.

François Leveque écrivait: "La mer a gagné sur la terre tout le long de ce Golfe depuis que se sont répandus les sables des Landes. La mer dévore le continent et la lecture des cartes marines permet de retrouver l'emplacement d'anciennes lignes de rivages...".

Welsch de son côté indiquait "L'invasion de cette partie du continent par les sables et le recul du littoral sous l'action de l'érosion marine sont deux faits en étroite interdépendance...".

A. Fabre rappelait "Les faits prouvent que la ligne de rivage dans cette partie du littoral n'est pas fixe, que la mer n'a cessé d'empiéter sur le continent depuis l'époque néolithique, qu'elle a encore gagné depuis l'époque romaine et qu'elle continue aujourd'hui son avance pendant les phases de désensablement du littoral..."

Dulignon-Desgranges estimait l'ancien rivage éloigné de $7 \mathrm{~km}$ du rivage actuel "avec une régression moyenne de $2 \mathrm{~m}$ par an...".

Se basant sur la découverte après chaque tempête de souches de pins, de chênes, de bruyères anciennes sur l'estran, P. Buffaut admet que ces arbres n'ont pu croittre et atteindre les dimensions qu'à grande distance de l'océan et seulement à l'abri des marées et des embruns.

Sans remonter aussi loin dans le temps, on a pu mesurer au cours du dernier siècle un recul non négligeable du littoral de Gascogne qui a obligé les collectivités locales ou le Ministère de l'Equipement à entreprendre des travaux de protection dans les secteurs les plus menacés.

A la Pointe de Grave, les travaux de défense entrepris sont le reflet des difficultés rencontrées pour lutter contre les actions marines, et il faut rappeler qu'entre 1825 et 1853 , la côte a reculé de $350 \mathrm{~m}$ entre la Pointe de Grave et la Pointe de la Negade emportant le Fort de Grave (Figure 8).

Entre 1785 et l'époque actuelle les relevés du trait de côte montrent un recul de plus de $2000 \mathrm{~m}$ à la Pointe de Grave et de plus de $500 \mathrm{~m}$ devant Soulac. Grâce aux nombreux ouvrages de protection il semble que le littoral se soit stabilisé dans ce secteur extrême Nord du Golfe.

Le même problème se retrouve d'ailleurs le long de la côte d'Arvert au Nord immédiat de l'estuaire girondin où le phare a dû être reconstruit très à l'intérieur des terres. Au cours de la dernière année, on a pu constater qu'en une seule tempête la côte pouvait reculer d'une dizaine de mètres entraînant une dégradation de la forêt domaniale.

A Montalivet le recul du rivage a été de $46 \mathrm{~m}$ entre 1875 et 1927 soit près de $0,90 \mathrm{~m}$ par an (P. Buffaut) certaines années la régression du rivage a pu atteindre 2 à $3 \mathrm{~m}$.

Au droit du Bassin d'Arcachon, les modifications du rivage sont encore plus sensibles et l'on cite des reculs de $10 \mathrm{~m}$ par an entre 1926 et 1936 . Il faut toutefois, dans cette partie du littoral, rappeler que les érosions spectaculaires de la côte du Pilat sont dues en grande partie à l'avancée de la Pointe du Cap Ferret qui renvoie les courants de remplissage et de vidage du bassin de plus en plus vers le Sud (Figure 9).

En 1907 Bouquet de la Grye signalait que d'après les mesures prises par le Service des Phares la mer avait gagné par an $0,31 \mathrm{~m}$ à Hourtin, $1,80 \mathrm{~m}$ à Contis, $1,52 \mathrm{~m}$ à Uchet...

A Capbreton d'après M. de Rouville "le recul général de la côte se traduirait par un déplacement de la laisse de basse-mer de $700 \mathrm{~m}$ au Nord et de $800 \mathrm{~m}$ au Sud entre 1881 et 1922 ; c'est à quelques distances au Sud 


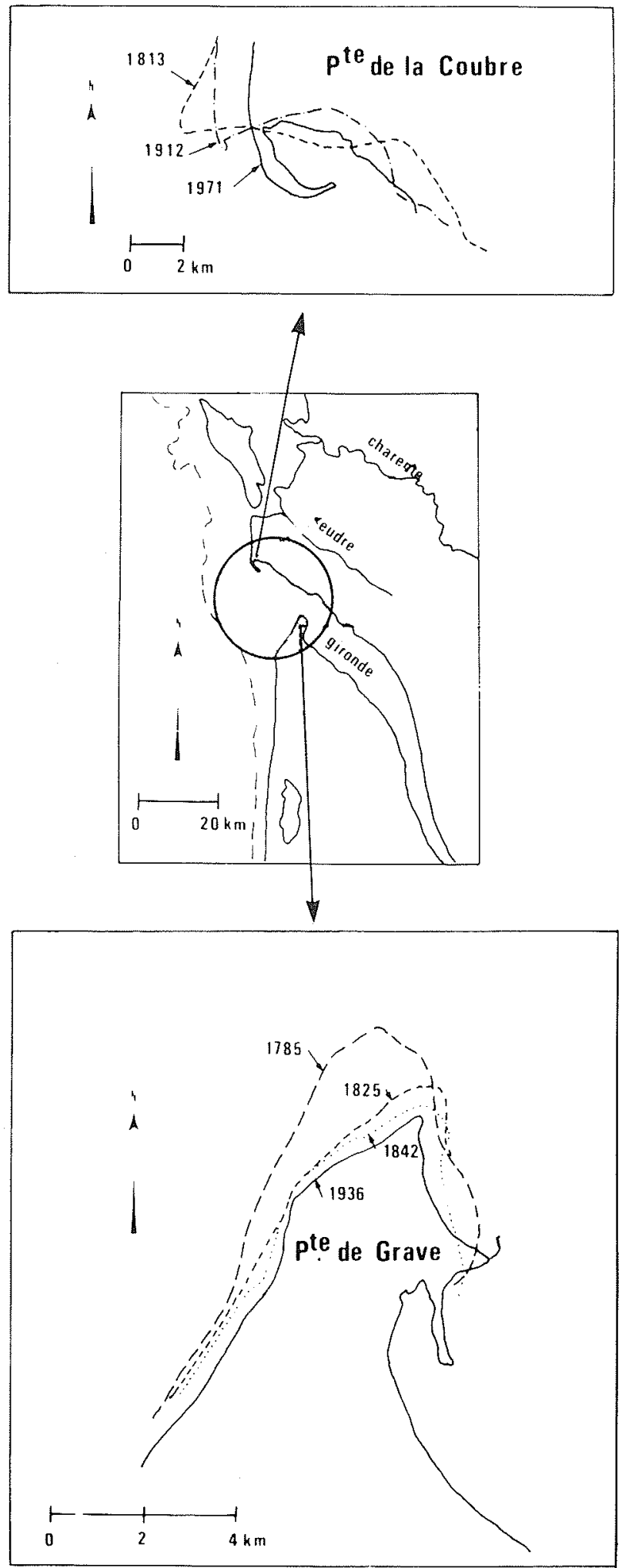

Figure 8 - Recul du littoral à la pointe de la Coubre et à la pointe de Grave
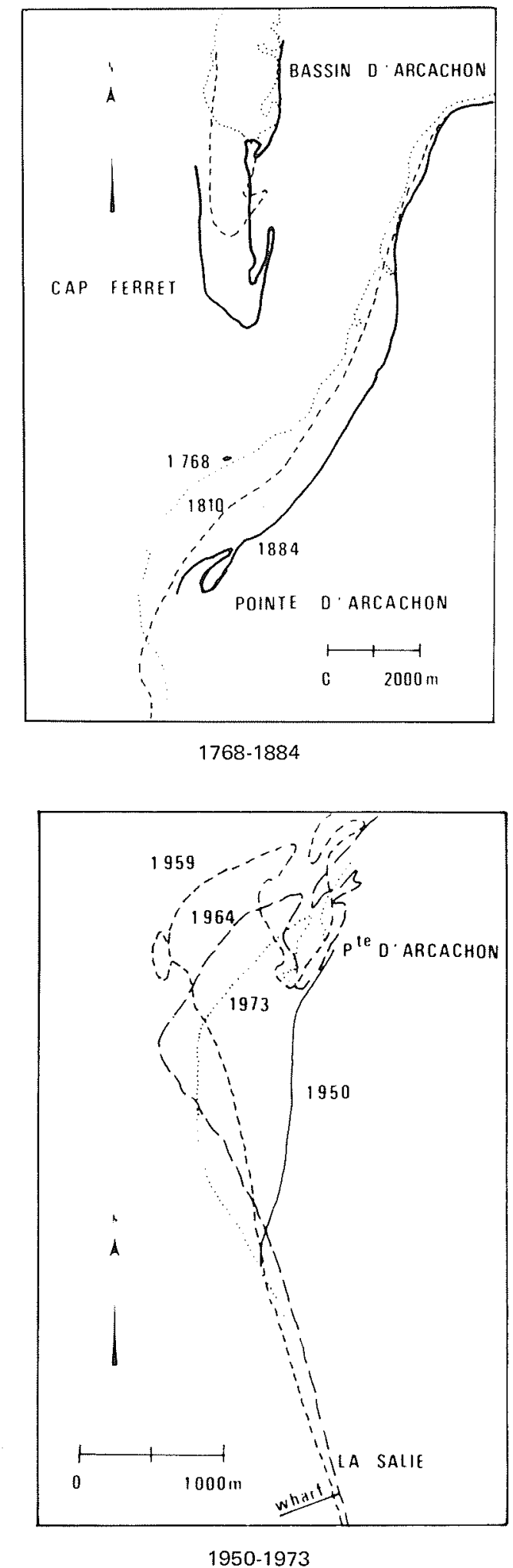

Figure 9 - Evolution du littoral à l'entrée du Bassin d'Arcachon 
des ouvrages de l'entrée que se sont produites des démolitions de villas au début de 1939, dans des conditions assez retentissantes"... Egalement, un peu après 1950 , la ligne de côte s'est brusquement érodée au cours d'une tempête et les villas situées en front de mer à Hossegor ont été partiellement détruites. Récemment l'extrémité de la digue de Cap Breton a été littéralement "engloutie" dans une fosse de -11 à $-14 \mathrm{~m}$ qui s'est produite au cours d'une tempête dans une zone où les fonds étaient à $-3 \mathrm{~m}$. On peut penser qu'une partie des sédiments du transit littoral se trouve dispersée dans le Gouf de Capbreton où les profondeurs de $-50 \mathrm{~m}$ apparaissent à $600 \mathrm{~m}$ seulement du littoral.

L'embouchure de l'Adour est un point singulier et de transition entre la côte landaise et la côte des Basques. Les courants de remplissage et de vidage de l'Adour, ainsi que les courants de crue forment un "épi hydraulique" qui arrête une partie du transit et le disperse vers le large formant avant réalisation des ouvrages de protection et des dragages une barre qui pouvait atteindre $-1,5 \mathrm{~m}$ en hiver. Le caprice de l'Adour qui se jetait en mer au Vieux Boucau il y a 400 ans a été barré à cette époque par Louis de Foix pour obtenir une embouchure rectiligne face à Bayonne mais il a fallu l'endiguer au XVIII ${ }^{\mathrm{e}}$ siècle pour éviter que, comme tous les courants landais, elle ne se porte vers le Sud et vienne se jeter à la Chambre d'Amour.

Endigage, dragage, digue de calibrage sur sa face Nord allaient permettre de faire passer les profondeurs d'accès au Port de Bayonne de $-1,50$ à $-10 \mathrm{~m}$ sous le zéro hydrographique.

L'érosion du littoral aux abords de l'Adour n'est pas le seul reflet de ces travaux d'aménagement indis. pensables pour l'avenir du Port. On constatait déjà entre 1826 et 1953, en comparant les plans de sondages du Service Hydrographique de la Marine, que les fonds devant toute la grande plage d'Anglet qui s'étend sur 5 kilomètres au Sud de l'Adour, s'étaient érodés de 3 à $4 m$ dans les petits fonds de -7 à $-3 \mathrm{~m}$ et que 15 millions de $m^{3}$ de sable avaient disparu de ce secteur bien avant la construction de la digue Nord. $\mathrm{Au}$ Nord de l'Adour par contre le trait de côte aurait été en légère sédimentation, une partie des matériaux en transit le long du littoral étant arrêtée par les petites jetées d'endiguement et par l'épi hydraulique de l' $\Lambda$ dour.

La comparaison de sondages du 16 Septembre 1898 , du 12 Septembre 1912 et du 27 Août 1953 confirme cette érosion du littoral d'Anglet avec un recul moyen annuel de 0,70 à $0,75 \mathrm{~m}$ par an. Ce phénomène semble s'être intensifié entre 1952 et 1963 où la côte a reculé de $35 \mathrm{~m}$ à $2 \mathrm{~km}$ au Sud de l'embouchure soit près de 3 m par an.

Plus au Sud on retrouve ce phénomène d'érosion et tous les anciens de Biarritz se souviennent de l'érosion de la grande plage de la côte des Basques où l'on ne retrouve plus maintenant qu'une faible épaisseur de sable de quelques dizaines de centimètres recouvrant les marnes ou les roches. On peut estimer que le niveau de la plage s'est abaissé de $2 \mathrm{~m}$ environ, les ouvrages réfléchissants de protection de la route ayant leur part de responsabilité.

N'étant plus freinées par les fonds sableux les vagues viennent attaquer à pleine mer le pied des falaises et s'associent aux phénomènes météorologiques de ravinement, infiltration, gel, vent, embruns pour détruire les falaises et avec elles les constructions qui s'élèvent à leur sommet. On peut estimer qu'avec un recul moyen de 0,60 $\mathrm{m}$ par an les falaises de la côte des Basques perdent, chaque année, 50000 à $100000 \mathrm{~m}^{3}$ de matériaux.

En descendant encore vers la frontière d'Espagne, on retrouve partout ce phénomène d'érosion et $M$. de Rouville indique que "les falaises argileuses de Guétary sont sapées par l'érosion à une cadence qui peut atteindre $0,50 \mathrm{~m} / \mathrm{an}$ ".

A Saint-Jean-de-Luz, d'après Quinette de Rochemont (cours de Travaux Maritimes - Tome II - Librairie Polytechnique) et jusqu'à la construction du briselames "le fond de la baie était fortement érodé, une partie de la ville bâtie le long de la mer sur le rivage sableux a été détruite. De la comparaison des plans de sondages dressés à diverses époques, il résulte que la laisse de basse-mer a reculé vers l'intérieur des terres de $0,90 \mathrm{~m} / \mathrm{an}$ à $2,65 \mathrm{~m} / \mathrm{an}$, le plus fort recul étant observé entre 1864 et 1873 . Le levé de 1864, comparé à celui de 1826 montre d'ailleurs que si la laisse des eaux a reculé durant ce temps de plus de $1 \mathrm{~m}$ par an, les lignes de profondeurs de 6 à $10 \mathrm{~m}$ ont reculé plus vite encore $(5 \mathrm{~m} / \mathrm{an}$ pour la ligne $-6 \mathrm{~m}$ entre 1826 et 1864 et $8 \mathrm{~m} /$ an entre 1864 et 1873 ), et que en même temps le talus de sable qui s'étend jusqu'aux grands fonds approchait, il se raidissait pour devenir encore plus menaçant"... (Figure 10).

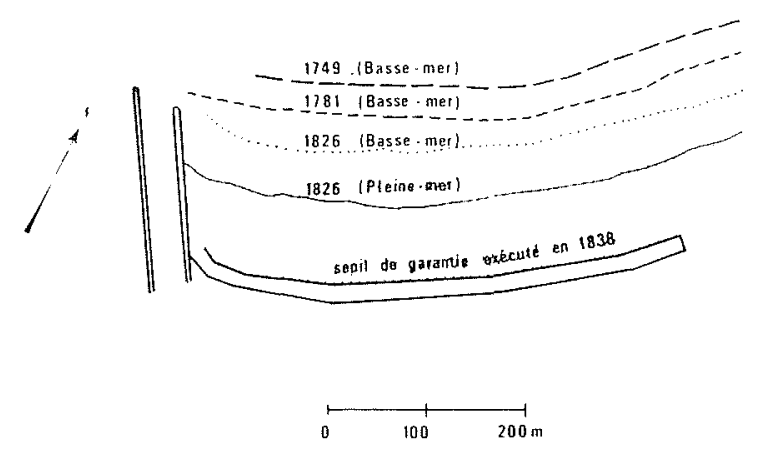

Figure 10 - Erosion de la plage de St Jean de Luz

Dans l'état actuel, bien que les digues existent, le fond de la baie a continué de s'éroder, nécessitant d'entreprendre des travaux de défense de la plage.

$A$ Hendaye, toujours d'après M. de Rouville, "les variations de la plage sont importantes et le recul de l'ordre de $0,50 \mathrm{~m}$ en moyenne à $2 \mathrm{~m} / \mathrm{an}$ en certains points critiques". Il a fallu réaliser un ouvrage important pour arrêter le phénomène et obtenir une sédimentation spectaculaire.

Quelle que soit la cause de l'érosion marine du littoral du Golfe de Gascogne un fait est certain, c'est qu'elle est générale depuis deux siècles et atteint des valeurs comprises entre 1 et 3 par an le long des zones sablonneuses et 0,5 à $0,6 \mathrm{~m}$ par an le long des falaises. Ces érosions ne sont pas constantes dans le temps et peuvent se stabiliser à certaines époques ou s'intensifier à d'autres suivant l'état de la mer et le déplacement 


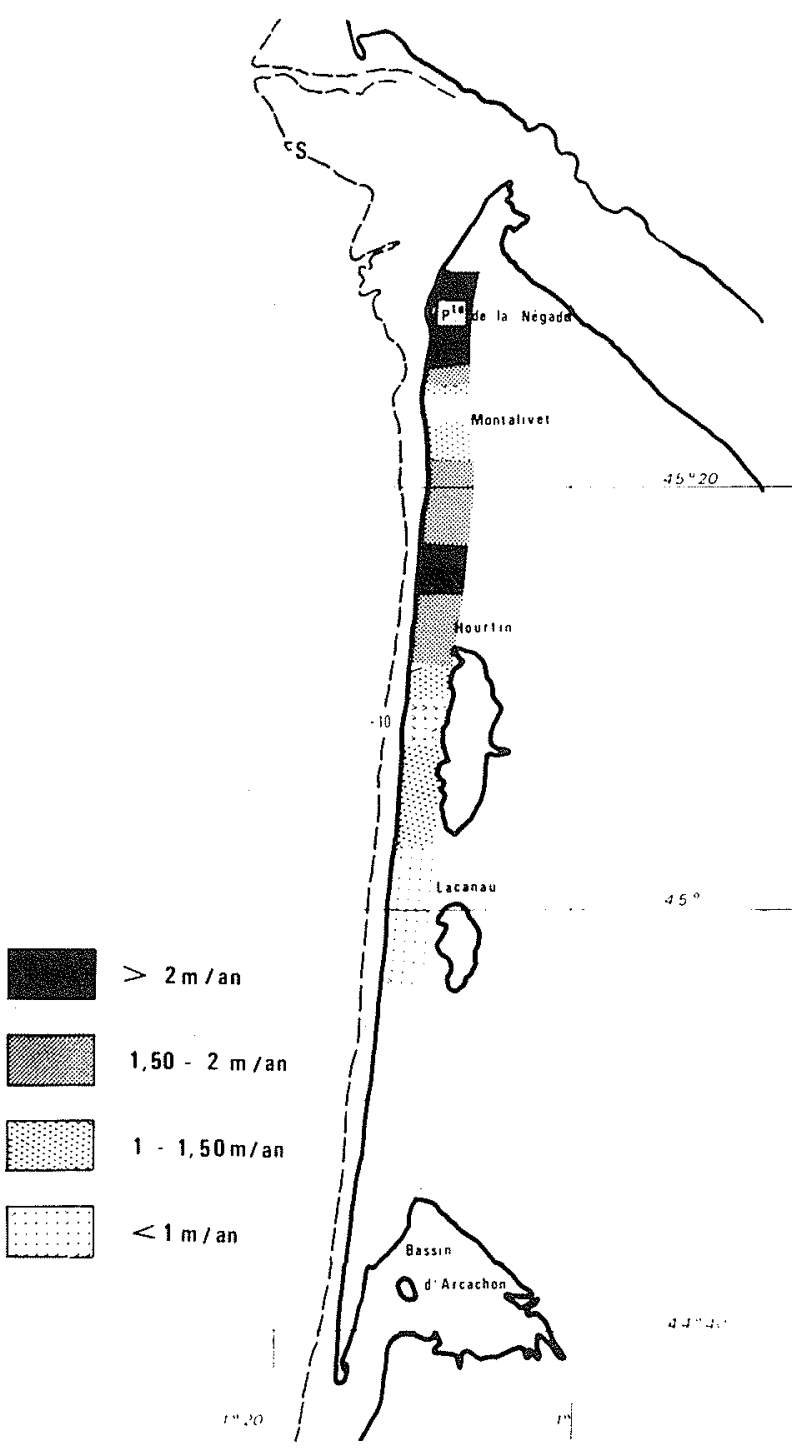

Figure 11 - Recul moyen du littoral de la côte des Landes

des stocks sédimentaires parallèlement au littoral (Figure 11).

Par suite d'une occupation plus importante de la zone côtière, ces aspects des mouvements sédimentaires ont pris une amplcur plus grande ct personnalisée pour telle ou telle partie du littoral. On retrouve dans le Golfe de Gascogne les phénomènes d'érosion générale qui existent aussi bien en Seine Maritime, le long du pays de Caux que sur les grandes plages du Cotentin ou de la Vendée.

\section{Le recul du littoral au cours des toutes dernières années}

On voudrait être optimiste pour ce recul ancestral du littoral du Golfe de Gascogne et penser que les documents antérieurs ne donnent pas toute la véracité des phénomènes, que dans notre $\mathrm{XX}^{\mathrm{e}}$ siècle l'homme a pu supprimer une érosion inéluctable.

Un examen détaillé du littoral du Golfe de Gascogne, les craintes des riverains, les dégradations que l'on cons- tate, montrent que si les services publics ont pu en certains points freiner les phénomènes d'érosion ils restent très importants dans les zones non protégées.

Quelques vues aériennes prises au début de l'automne 1978 permettent d'illustrer les évolutions du littoral et d'en apprécier la morphologie générale.

De la Pointe de Grave à Soulac la côte est relativement stable, de grandes accumulations apparaissant au Nord de la Pointe Saint Nicolas.

A Soulac on commence à trouver à $2 \mathrm{~km}$ au Sud des traces d'érosion, les blockhaus réalisés pendant la guerre étant submergés (photographie 12). Toute l'ampleur du phénomène se situe à l'Amélie où le recul du littoral a pu atteindre certaines années jusqu'à $20 \mathrm{~m}$ par an ; entre 1940 et 1970 le haut estran limité par les dunes a reculé de $200 \mathrm{~m}$ emportant successivement le Boulevard de l'Océan, la rue des Olives et venant tangenter la route de Soulac (photographie 13). Les blockhaus sont maintenant très loin en mer alors qu'ils étaient initialement entre 50 et $100 \mathrm{~m}$ à l'intérieur des dunes (photographie 14); certains sont en mer à $185 \mathrm{~m} \mathrm{du}$ rivage actuel.

La Pointe de la Negade n'échappe pas à cette érosion et l'on trouve au Gurp les vestiges allemands éboulés sur l'estran (photographie 15) avec une microfalaise abrupte en haut de plage.

A Montalivet (photographie 16), le phénomène d'érosion semble moins important qu'au Nord. Ce fait

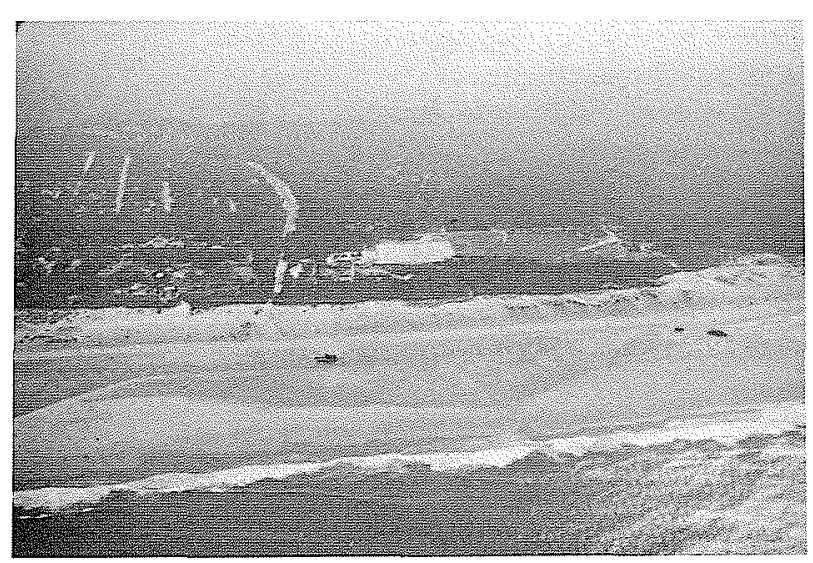

Figure 12 - Erosion du littoral au Sud de Soulac

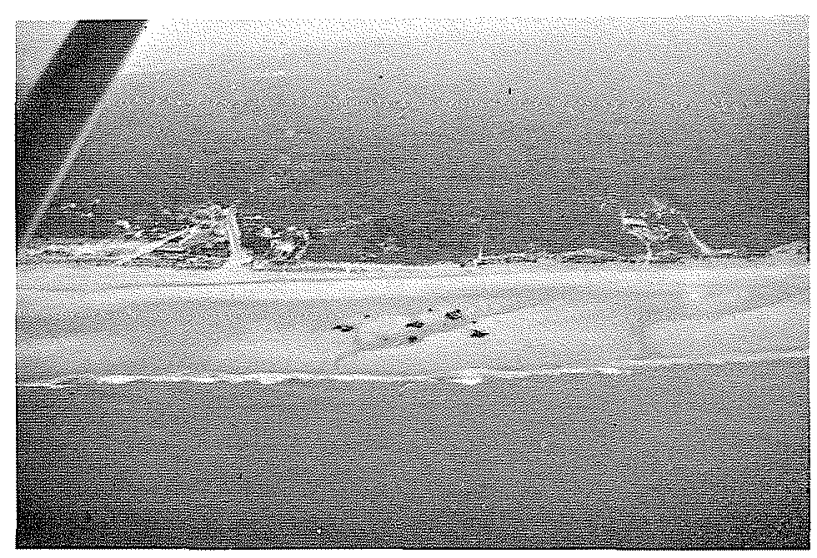

Figure 13 - Erosion à l'Amélie 


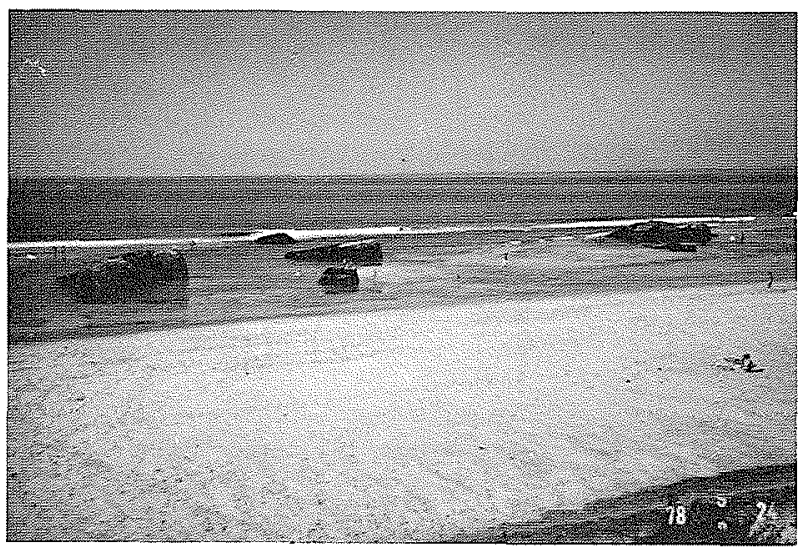

Figure 14 - Disparition en mer des blockhaus de l'Amélie

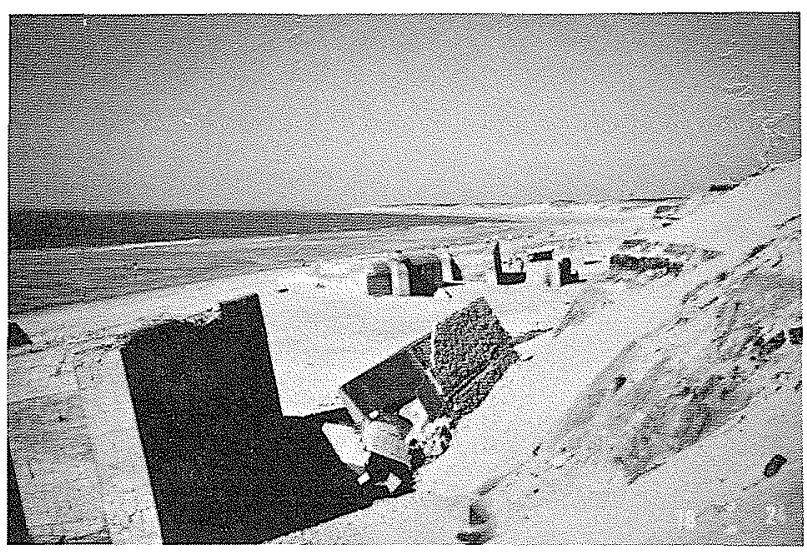

Figure 15 - Erosion du Gurp (pointe de la Négade)

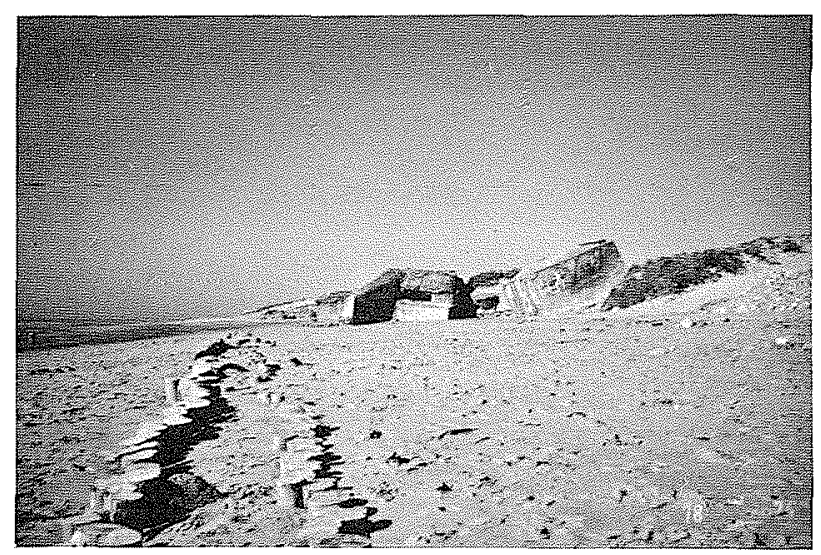

Figure 16 - Plage de Montalivet protégée par des pieux en bois

peut être dû à la présence à mi-estran d'un filtre constitué par deux rangées de pieux battus distantes de $0,60 \mathrm{~m}$ et comblé de galets.

A Hourtin on retrouve l'attaque par la mer des dunes littorales, le niveau de l'estran s'est abaissé et les vestiges terrestres de 1942 apparaissent sur la plage (Photographie 17). Le recul du littoral atteint $2 \mathrm{~m} / \mathrm{an}$ devant la forêt du Junca et $1,60 \mathrm{~m}$ devant Hourtin.

$A$ Carcans plage, c'est-à-dire à mi-chemin entre la

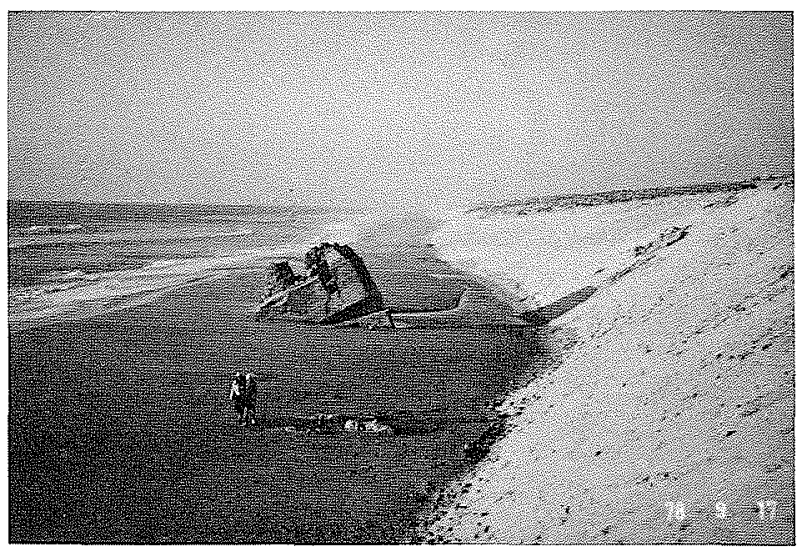

Figure 17 - Erosion à Hourtin

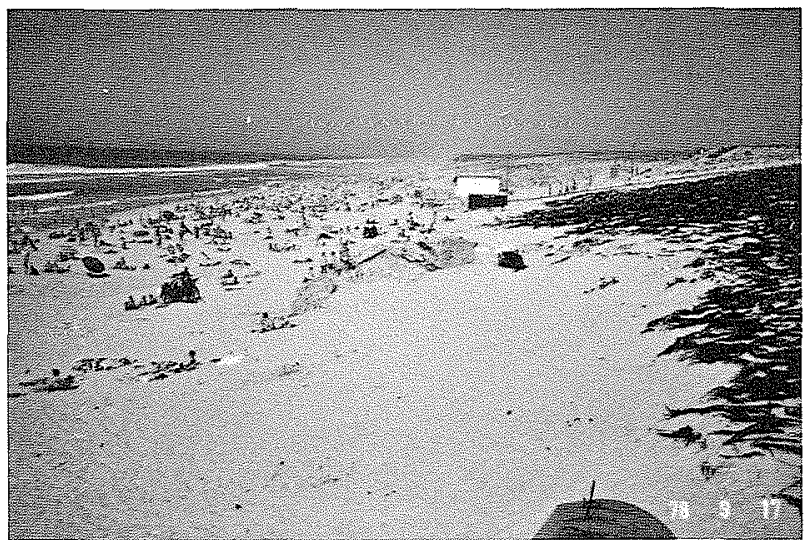

Figure 18 - La plage de Le Porge

Pointe de Grave et le Bassin d'Arcachon on retrouve les microfalaises d'érosion et les vestiges d'anciens ouvrages. Le recul moyen "annuel serait de l'ordre du mètre.

A Lacanau si l'érosion persiste elle est un peu moins sensible dans l'état actuel et atteint $0,60 \mathrm{~m} / \mathrm{an}$.

De même à Le Porge (photographie 18) le profil de plage est plus uniforme et ne présente pas de microfalaise abrupte comme dans d'autres parties de la côte des Landes, l'Office National des Forêts ayant pu d'ailleurs intervenir pour adoucir la pente de la dune et la régler à moins de $20 \%$

Entre le sémaphore et le phare du Cap Ferret le recul du trait de côte est important et concerne un secteur de falaises soumises à des éboulements considérables.

D'une façon très générale on peut dire qu'entre Montalivet et Cap Ferret les érosions diminuent progressivement du Nord au Sud passant de $3 \mathrm{~m}$ par an environ à $0,50 \mathrm{~m}$ par an. Dans l'état actuel il semble que l'érosion gagne vers le Sud.

Le Cap Ferret et l'entrée du Bassin d'Arcachon forment une entité spéciale avec l'avancée spectaculaire de la flèche du Ferret, les déplacements alternatifs des passes, les érosions des dunes du Pilat. Houles et courants s'allient pour façonner ce secteur, l'éroder ou le 


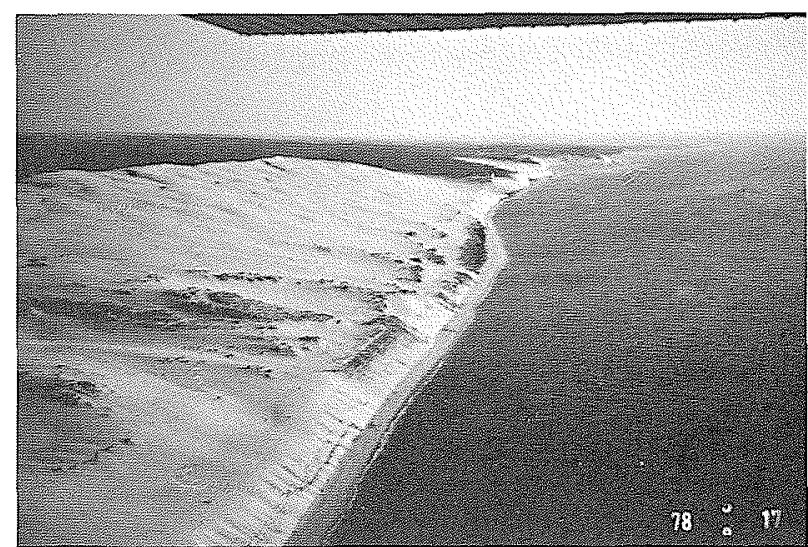

Figure 19 - Erosion des dunes du Pilat

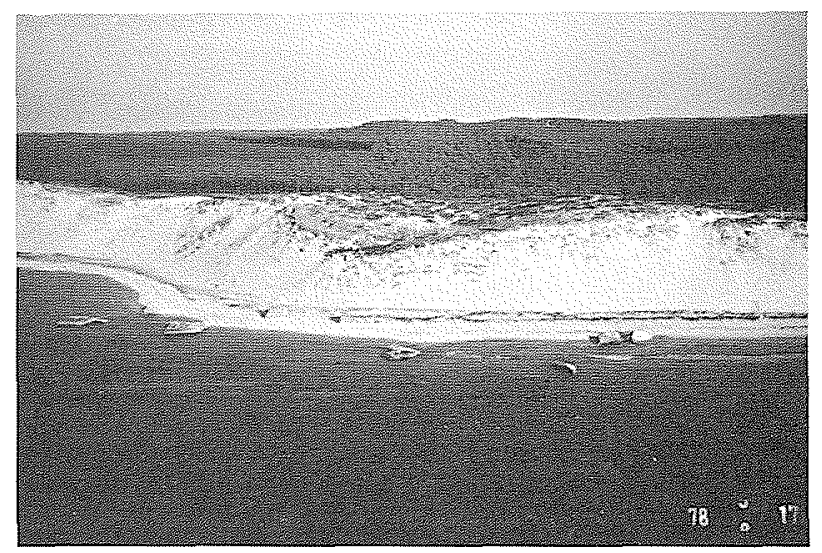

Figure 20 - Pointe d'Arcachon (Pointe de Gaillouneys)

sédimenter libérant périodiquement des masses considérables de sédiments qui se trouveront disponibles pour transiter vers le Sud (Photographie 19).

A la pointe d'Arcachon la falaise est verticale, les blockhaus sont très loin en mer accusant un violent recul du littoral (photographie 20 prise à la Pointe de Gaillouneys).

A Biscarosse des maisons sont à la limite de la pleine mer et la côte s'étend ensuite régulièrement jusqu'à Mimizan tout le long de la zone militaire avec un recul assez faible du littoral (0,50 $\mathrm{m}$ par an environ).

$A$ Mimizan des épis ont été implantés pour protéger la plage et la sortie du courant a été canalisée. La côte est relativement stable.

$A$ Contis la canalisation du courant a provoqué une sédimentation au Nord et une érosion au Sud (photographie 21), on retrouve les blockhaus en mer accusant une érosion appréciable au Sud du courant.

La côte est relativement stable de Contis à St Girons et l'on ne peut qu'espérer qu'elle le restera pour préserver la ville de St Girons Plage qui s'est installée à l'emplacement de la dune.

A Moliets le courant est dévié vers le Sud par les courants de houle, passant du Huchet à Miolets $(3 \mathrm{~km})$. $\mathrm{Ce}$ courant forme un épi hydraulique qui donne un déficit sédimentaire au Sud où une érosion apparaît très localement.

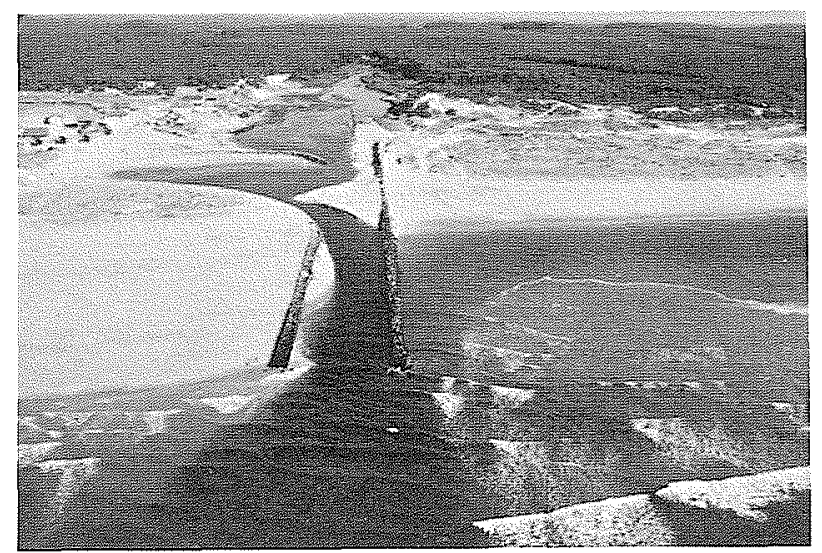

Figure 21 - Accumulation des sables au Nord du courant de Contis

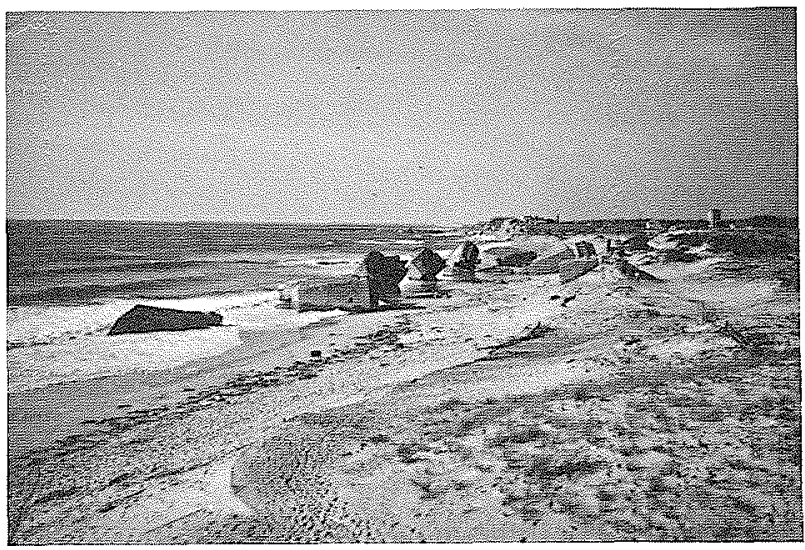

Figure 22 - Destruction du littoral à Cap Breton

Au Sud de Moliets de la madrague apparaît en quantité notable sur la plage permettant de penser que jadis, avant l'an 1500, 1'Adour se jetait au voisinage de cette zone et a déposé vers le large des éléments grossiers.

Au Vieux Boucau la plage a reculé et des maisons qui étaient à $50 \mathrm{~m}$ à l'intérieur du haut estran sont maintenant menacées.

Si Hossegor a été très érodé avant la construction de la jetée Nord du courant et de la sortie au Lac d'Hossegor, il jouit actuellement d'une certaine stabilité.

Par contre au Sud, à Capbreton le déficit du transit littoral a provoqué un recul de la côte catastrophique malgré les ouvrages de protection (Photographie 22).

On retrouve cette forte érosion à Labenne, à Ondres, à Metro (Photographie 23) et jusqu'à l'embouchure de l'Adour. Certaines années le recul du littoral a atteint $10 \mathrm{~m}$ par an.

Au Sud de l'Adour, devant Anglet, les interventions humaines ont encore augmenté les phénomènes d'érosion et le recul du littoral qui atteignait 3 à $4 \mathrm{~m}$ par an avant la construction de la digue Nord, représentant un volume d'érosion de $200000 \mathrm{~m}^{3}$ par an, a pu atteindre $10 \mathrm{~m}$ par an au cours d'années exceptionnelles, la moyenne du recul au cours des 20 dernières années étant d'une centaine de mètres soit $5 \mathrm{~m}$ par an. Le volume total d'érosion annuelle avait été évalué, entre 1963 et 1973, à $300000 \mathrm{~m}^{3}$ par an. En 1973 le niveau 


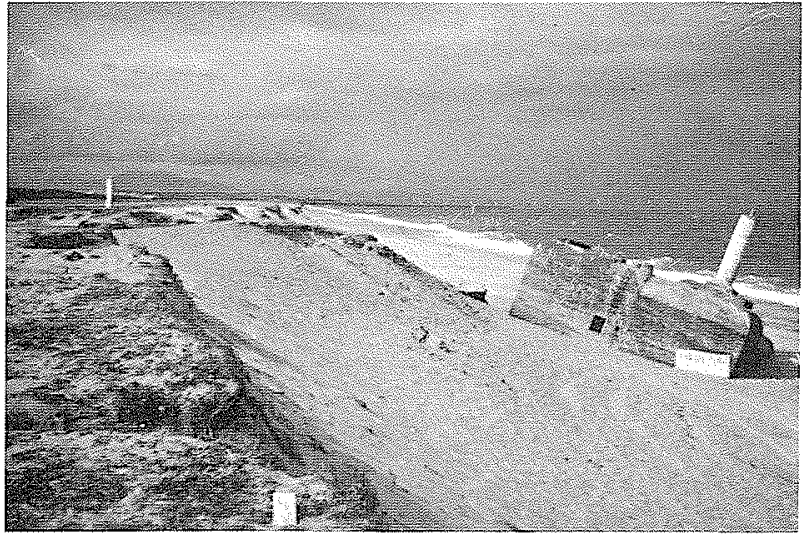

Figure 23 - Erosion du littoral à Metro

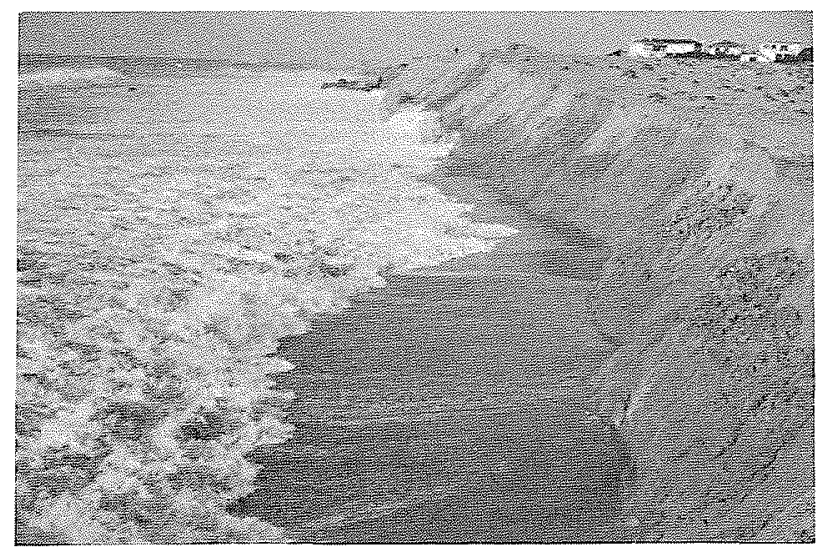

Figure 24 - Erosion du littoral au Nord de Chiberta en 1973.

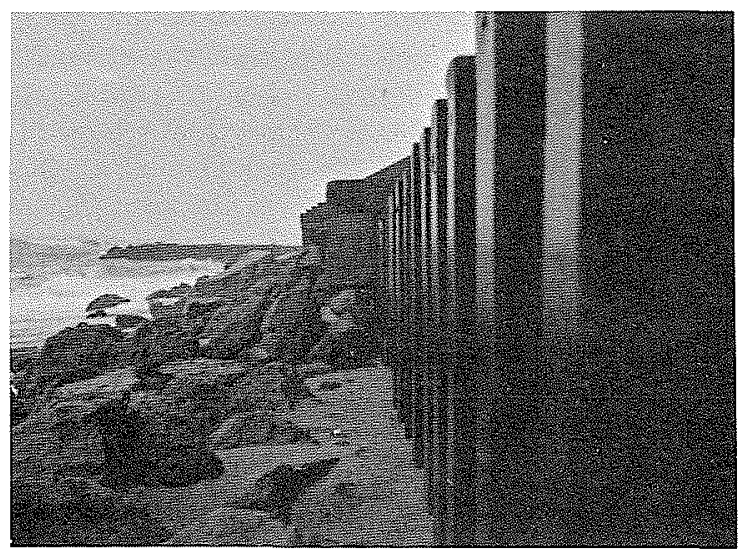

Figure 25 - Destruction du mur des Sables d'Or et mise à nu des palplanches (1973).

des plages devant Anglet-Chiberta avait baissé de plusieurs mètres formant une véritable falaise accore avec la côte (Photographie 24). Devant les Sables d'Or le mur de protection était partiellement détruit, les palplanches étant déchaussées (Photographie 25) tandis qu'au Nord il ne restait plus que des ruines de l'ancien ouvrage de protection de la route littorale (Photographie 26) et qu'au Sud les installations de V.V.F. étaient menacées, le parking entièrement creusé avec une dénivellation

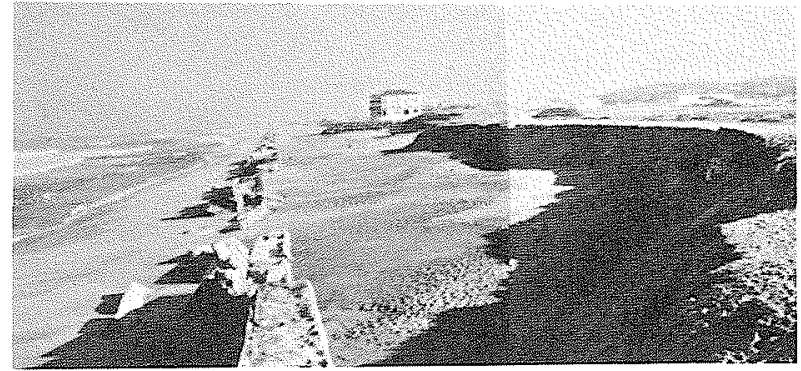

Figure 26 - Destruction du mur de protection de la route littorale à Anglet (1973)

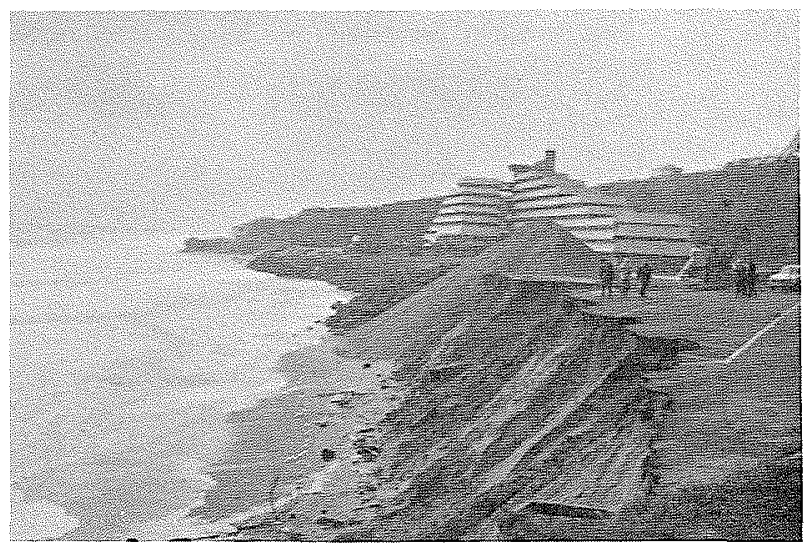

Figure 27 - Erosion de la plage devant le V.V.F. d'Anglet (1973).

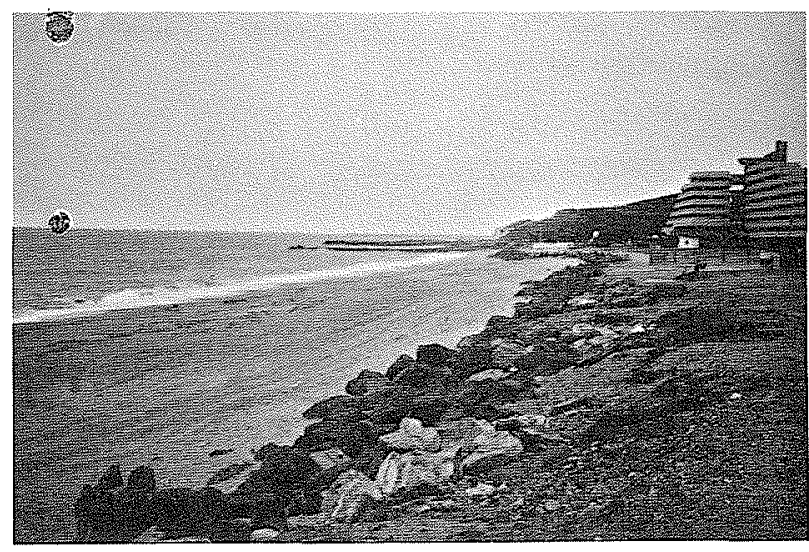

Figure 28 - Engraissement des fonds devant le V.V.F. après les travaux de protection en 1977

de plus de 10 mètres avec le niveau du haut de plage (Photographie 27).

Il a fallu les importants travaux engagés par la Ville d'Anglet ces dernières années pour freiner une érosion catastrophique pour les installations riveraines et assurer une certaine stabilité de la plage dans la zone protégée comme on peut le constater sur les vues prises récemment devant le V.V.F. (Photographie 28) et au Nord immédiat des Sables d'Or (Photographies 29 et 30).

Cette solution d'épis n'est pas très esthétique, elle est chère, demande un entretien, mais a permis locale- 


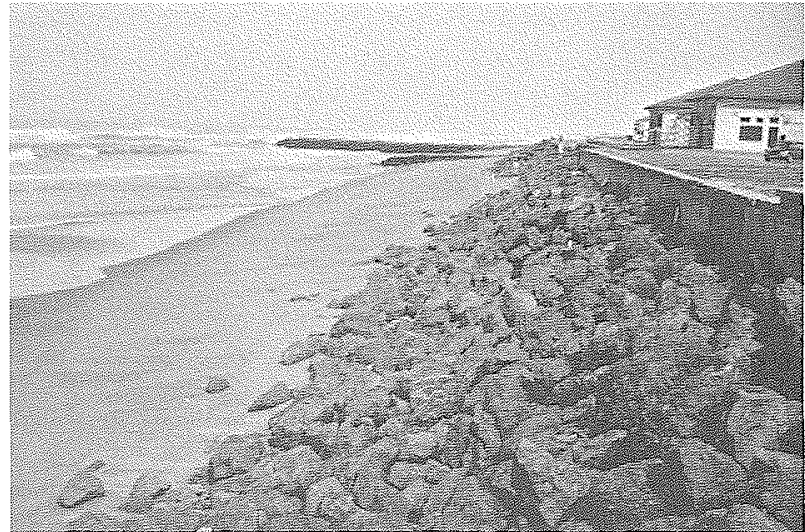

Figure 29 - Engraissement des plages de Chiberta après réalisation des épis en 1977

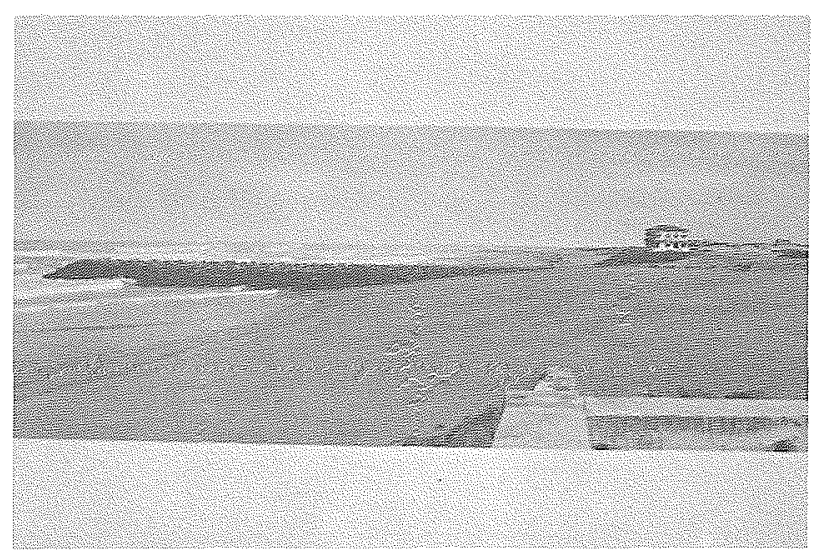

Figure 30 - Reformation de la plage devant les Sables d'Or après réalisation des épis en 1978 .

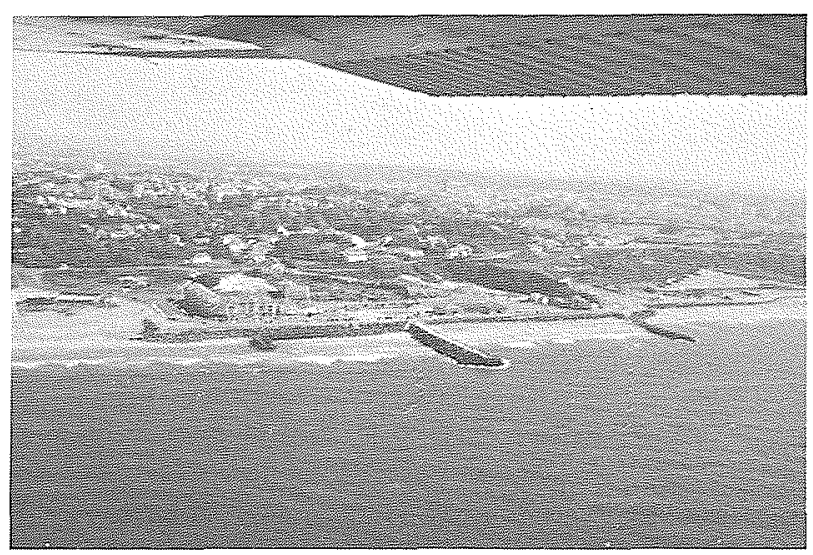

Figure 31 - Vue générale de la plage d'Anglet protégée par des épis en 1978

ment de freiner ou de supprimer un phénomène d'érosion inexorable qui s'avèrait catastrophique. Actuellement si ces travaux n'avaient pas été entrepris tout l'ilot urbanisé situé au centre et au Sud de la côte d'Anglet aurait disparu alors que les plages continuent de persister et les petits fonds de s'exhausser devant les ouvrages (Photographie 31).

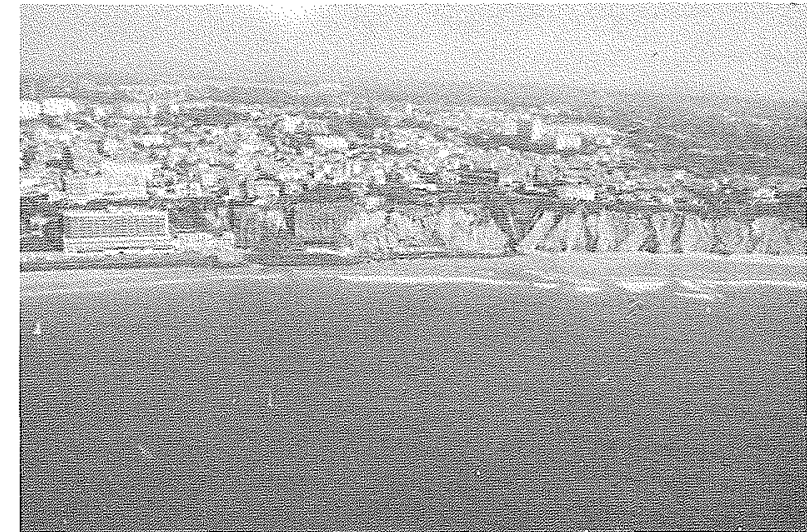

Figure 32 - Vue générale des falaises de la côte des Basques

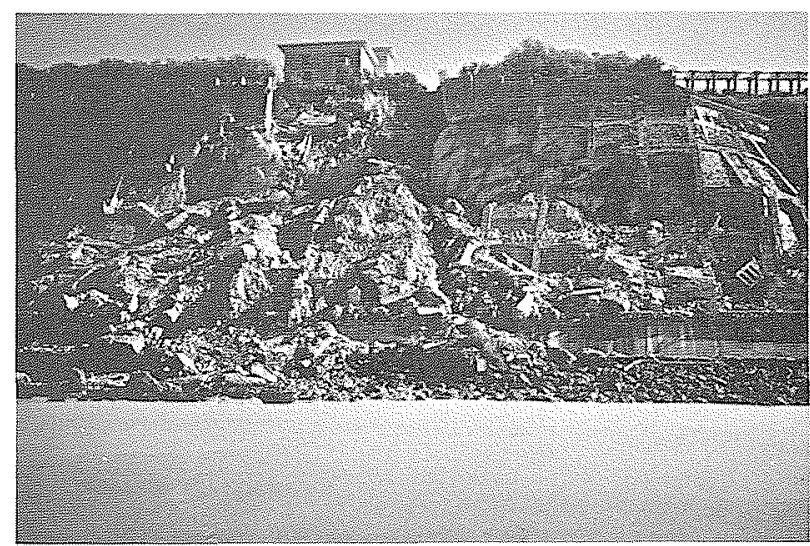

Figure 33 - Destruction des falaises de Biarritz.

En arrivant sur Biarritz les falaises remplacent les dunes mais les érosions continuent sous l'attaque implacable des vagues et l'infiltration des eaux dans les terres. Le recul du littoral se maintient avec 0,50 à $0,60 \mathrm{~m} / \mathrm{an}$ et les plages s'appauvrissent (photographie 32 et photographie 33). Le même phénomène se continue vers Hendaye et la "pile d'assiettes" que l'on se plaisait à faire admirer aux touristes a disparu.

St Jean de Luz que l'on croyait enfin protégée par les puissants ouvrages réalisés à l'entrée de sa baie voit elle-même sa plage se dégarnir nécessitant des travaux de rechargement et de protection.

\section{Conclusions}

Tous ces exemples de l'évolution du littoral du Golfe de Gascogne, de l'embouchure de la Gironde à la frontière d'Espagne, montrent combien l'équilibre de cette côte est précaire et l'importance de son recul au cours des temps.

Recul sous l'action de phénomènes géologiques inéluctables au cours de la surélévation du niveau des eaux à l'époque de la transgression flandrienne, recul depuis plus de deux siècles sous l'action des houles, recul à l'époque actuelle par suite d'un épuisement du stock sédimentaire disponible, de la puissance exceptionnelle des vagues, de certaines interventions humaines. 
Ce recul a pu atteindre à certaines époques $10 \mathrm{~m}$ par an à la Pointe de Grave. Devant l'Amélie, il a été en moyenne de $7 \mathrm{~m}$ par an au cours des 30 dernières années avec des reculs brutaux de $20 \mathrm{~m}$ au cours d'années exceptionnelles. A Montalivet il est de $3 \mathrm{~m}$ par an et décroît progressivement jusqu'à $0,50 \mathrm{~m}$ par an au Cap Ferret. Les dunes du Pilat, de la Pointe de Gaillouneys, restent un point de grande érosion et si le littoral est moins attaqué jusqu'au Vieux Boucau on rencontre au Sud de Capbreton de très violentes érosions avec, certaines années, des reculs de $10 \mathrm{~m}$.

La côte d'Anglet, premier maillon de la Côte des Basques, n'est pas épargnée et les dégâts récents mon- trent toute l'ampleur du phénomène qui a pu atteindre $15 \mathrm{~m}$ d'érosion certaines années et que l'on a dû freiner à grands frais. Les falaises de Biarritz, Guétary, des abords de St Jean de Luz ne sont pas épargnées.

Presque partout ce littoral recule sous la puissance des vagues. Souhaitons que les hommes n'aggraveront pas la situation déjà précaire en effectuant des emprunts inconsidérés de sable ou des ouvrages qui perturbent fortement l'équilibre sédimentaire. Souhaitons aussi que dans leur sagesse les hommes tiennent compte de cette érosion inexorable en interdisant les constructions trop près du rivage et en réalisant des ouvrages de défense appropriés. 